\title{
Electro-osmotic flow and mixing in heterogeneous microchannels
}

\author{
Jin-bai Zhang, ${ }^{1,4}$ Guo-wei He, ${ }^{1,2, *}$ and Feng Liu ${ }^{3}$ \\ ${ }^{1}$ LNM, Institute of Mechanics, Chinese Academy of Sciences, Beijing, 100080, China \\ ${ }^{2}$ Department of Aerospace Engineering, Iowa State University, Ames, Iowa 50011-2271, USA \\ ${ }^{3}$ Department of Mechanical Aerospace Engineering, University of California, Irvine, California 92697-3975, USA \\ ${ }^{4}$ NLCFD, School of Aeronautic Science and Engineering, Beihang University, Beijing, 100083, China
}

(Received 24 September 2005; published 12 May 2006)

\begin{abstract}
Analytical and numerical studies of secondary electro-osmotic flow (EOF) and its mixing in microchannels with heterogeneous zeta potentials are carried out in the present work. The secondary EOFs are analyzed by solving the Stokes equation with heterogeneous slip velocity boundary conditions. The analytical results obtained are compared with the direct numerical simulation of the Navier-Stokes equations. The secondary EOFs could transport scalar in larger areas and increase the scalar gradients, which significantly improve the mixing rate of scalars. It is shown that the heterogeneous zeta potentials could generate complex flow patterns and be used to enhance scalar mixing.
\end{abstract}

DOI: 10.1103/PhysRevE.73.056305

PACS number(s): 47.65.-d, 82.70.-y, 66.10.-x

\section{INTRODUCTION}

Microfluidics has a broad range of applications in chemistry and biotechnology, for example, sample injection, drug delivering and solution mixing and separations [1]. All of these techniques require handling fluids in the low Reynolds number $(\mathrm{Re})$ regime, that is, manipulating the fluids moving in microdevices to achieve desired flow patterns. Conventional pressure-driven technology needs significant pressures that are difficult to deliver in microdevices. Moreover, the technique is difficult to generate complex flow patterns required for different purposes in biotechnology. Electroosmotic flow (EOF) [2] is an alternative to pressure-driven flows with convenient implementation. In this paper, we will investigate the secondary flows of EOFs in microchannels which could exhibit the complex flow patterns that offer opportunities for rapid mixing.

Electro-osmotic flow or electro-osmotics is the bulk movement of liquid near a stationary surface due to an externally applied electric field [2]. Most solid substances will acquire a relative electric charge when they are in contact with a liquid. Ions of opposite charge to that of the surfaces are attracted towards the surfaces, and ions of the same charge are repelled from the surfaces. The net effect is to form a thin layer of liquid close to the charged surfaces. The thin layer of liquid, which is composed predominately of ions of charge opposite to that of the charged surfaces, is called the electric double layer (EDL). If an external electric field is applied to EDL, an electrical body force is exerted on the excess of the ions of the opposite charge. The ions will be driven by the imposed electrical body force and then pull the liquid with them. The EDL moves along the charged surface acting as a slip layer at the Helmholtz-Smoluchowski velocity [2]. Due to viscous drag, the bulk flow will be driven by EDL and moves through microdevices, which leads to EOFs.

\footnotetext{
*Author to whom correspondence should be addressed. Electronic address: hgw@lnm.imech.ac.cn
}

The driving force for the EOF is dependent on the surface charge distributions and the applied electric fields. The interaction of the surface charge distributions with the EDL can be described by the zeta potential. Therefore, the EOF's behavior is dependent on the zeta potential. The variable zeta potentials generate different slip velocities that induce various flow patterns. The classic result is that the EOF moves as in plug flows under the uniform zeta potential [2]. Anderson and Idol found that a nonuniform zeta potential varying in the flow direction could induce symmetrical secondary flows [3]; Ghosal developed asymptotic solutions for EOFs with zeta potentials that slowly change with axial direction [4]; Herr et al. experimentally studied EOFs in a cylindrical capillary with a step change in the zeta potential [5]; Stroock et al. demonstrate that the variation of the zeta potential normal to the flow direction could generate mixing layers, while the sign variation along the flow direction could generate cellular flows [6]. Furthermore, we will study the flow patterns in microchannels with heterogeneous zeta potentials, where the potentials vary in both axial and lateral directions. This offers an opportunity to generate complex flow patterns for flow control in microfluidics.

The heterogeneous zeta potentials can be achieved through chemical modifications, coupled capillaries, and integrated systems. They can be used to generate secondary EOFs and thus enhance mixing $[7,8]$. The rapid mixing at microscales is important in many microfluidic systems $[9,10]$. However, the conventional mixing tools, such as turbulent mixing, are not available. [11] The EOFs provide a promising tool for rapid mixing at microscales. In many microfluidic devices, the microchannels have rectangle cross sections with large aspect ratios. The flows in the microchannels of large aspect ratios can be approximately treated as two dimensional ones. The present paper is devoted to twodimensional EOFs: in Sec. II, secondary EOFs will be investigated analytically; in Sec. III, we will numerically investigate EOF and its mixing; Sec. IV will be devoted to the concluding remarks. 


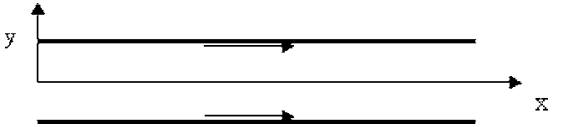

FIG. 1. A schematic diagram for a two-dimensional microchannel.

\section{THEORETICAL ANALYSIS OF EOFs}

We consider an electro-osmotic flow (EOF) through a two-dimensional microchannel of height $h=2 \delta$ and length $L$, where $L \gg \delta$ (for example, $\delta=1$ ). The EOF is confined by two flat insulating surfaces and moves along the microchannel. It can be described by reference to an orthogonal coordinate system with the $x$ axis in the streamwise direction and $y$ axis in the normal direction; see Fig. 1 for a schematic diagram of a two-dimensional microchannel EOF.

The EOFs obey the governing equation as follows:

$$
\begin{aligned}
\rho_{0}\left(\frac{\partial \mathbf{u}}{\partial t}+\mathbf{u} \cdot \nabla \mathbf{u}\right) & =-\nabla p+\mu \nabla^{2} \mathbf{u}, \\
\boldsymbol{\nabla} \cdot \mathbf{u} & =0
\end{aligned}
$$

with the nondimensional Helmholtz-Smoluchowski slip velocity boundary conditions [2]

$$
\begin{aligned}
& u(x,+1) \equiv u^{t}(x)=-\frac{\epsilon E}{\mu} \zeta^{t}(x), \\
& u(x,-1) \equiv u^{b}(x)=-\frac{\epsilon E}{\mu} \zeta^{b}(x),
\end{aligned}
$$

where $u(x, y)$ and $v(x, y)$ are the streamwise and normal components of velocity fields, respectively. Here, $\rho_{0}$ is the fluid density, $p$ the pressure, $\epsilon$ the dielectric constant, and $\mu$ the fluid viscosity. The slip velocities on the boundary are dependent on a constant electric field $E$ applied along the channel and a zeta potential $\zeta$ of the surface. The superscripts " $t$ " and " $b$ " represent the top and bottom flat surface. It is assumed that the electric double layers are thin compared to the half height of the channel $\delta$ and thus its effects can be simply described by the slip velocity boundary conditions (3). Therefore, zeta potentials and slip velocities have the same implications in the sense of the driving force for EOFs.

The simplest form of zeta potentials is homogeneous: uniform along the channel and equal on the two flat surfaces. The zeta potential leads to a uniform and symmetric slip velocity on the flat surfaces, which yields the plug flows. However, the zeta potentials are not necessarily homogeneous. Generally, they are heterogeneous: nonuniform along the channel and asymmetric between the two flat surfaces. The heterogeneous zeta potentials produce heterogeneous slip velocities that could yield complex EOFs.

For the two-dimensional EOF, a stream function $\psi(x, y)$ can be introduced,

$$
u(x, y)=\frac{\partial \psi(x, y)}{\partial y}, \quad v(x, y)=-\frac{\partial \psi(x, y)}{\partial x} .
$$

We will only consider the steady states of the EOF at a low Reynolds number. In this case, the governing equation for the stream function is

$$
\nabla^{4} \psi=0
$$

and the boundary conditions are

$$
\begin{gathered}
y=1: \psi=Q, \quad \frac{\partial \psi}{\partial y}=u^{t}(x), \\
y=-1: \psi=0, \quad \frac{\partial \psi}{\partial y}=u^{b}(x),
\end{gathered}
$$

where $Q$ is the volume flow flux. It can be obtained from the momentum equation in the axial direction

$$
Q=\left[\bar{u}_{0}^{t}+\bar{u}_{0}^{b}\right] \delta
$$

where

$$
\bar{u}_{0}^{t}=\frac{1}{L} \int_{0}^{L} u^{t}(x) d x, \quad \bar{u}_{0}^{b}=\frac{1}{L} \int_{0}^{L} u^{b}(x) d x .
$$

The Fourier series approach will be used to solve Eq. (5) with boundary conditions (6) and (7). We expand the stream function and the slip velocities with respect to $x$,

$$
\begin{gathered}
\psi(x, y)=\bar{\psi}_{0}(y)+2 \sum_{m=1}^{\infty} \hat{\psi}_{s, m}(y) \sin \left(\frac{2 \pi m x}{L}\right) \\
+2 \sum_{m=1}^{\infty} \hat{\psi}_{c, m}(y) \cos \left(\frac{2 \pi m x}{L}\right), \\
u^{t}(x)=\bar{u}_{0}^{t}+2 \sum_{m=1}^{\infty} \hat{u}_{s, m}^{t} \sin \left(\frac{2 \pi m x}{L}\right)+2 \sum_{m=1}^{\infty} \hat{u}_{c, m}^{t} \cos \left(\frac{2 \pi m x}{L}\right), \\
u^{b}(x)=\bar{u}_{0}^{b}+2 \sum_{m=1}^{\infty} \hat{u}_{s, m}^{b} \sin \left(\frac{2 \pi m x}{L}\right)+2 \sum_{m=1}^{\infty} \hat{u}_{c, m}^{b} \cos \left(\frac{2 \pi m x}{L}\right),
\end{gathered}
$$

where the Fourier coefficients for the stream function are given below:

$$
\begin{gathered}
\bar{\psi}_{0}(y)=\frac{1}{L} \int_{0}^{L} \psi(x, y) d x, \\
\hat{\psi}_{s, m}(y)=\frac{1}{L} \int_{0}^{L} \psi(x, y) \sin \left(\frac{2 \pi m x}{L}\right) d x, \\
\hat{\psi}_{c, m}(y)=\frac{1}{L} \int_{0}^{L} \psi(x, y) \cos \left(\frac{2 \pi m x}{L}\right) d x .
\end{gathered}
$$

The Fourier coefficients for the slip velocities can be defined in the same way as in Eq. (11). 
We substitute Eq. (10) into Eqs. (5)-(7) and compare the coefficients of each mode. The comparison yields the following equations for the coefficients of the Fourier modes:

(1) $m=0$

$$
\begin{gathered}
\frac{\partial^{4} \bar{\psi}_{0}}{\partial y^{4}}=0, \\
y=1: \bar{\psi}_{0}(y)=Q, \quad \frac{\partial \bar{\psi}_{0}}{\partial y}=\bar{u}^{t}, \\
y=-1: \bar{\psi}_{0}(y)=0, \quad \frac{\partial \bar{\psi}_{0}}{\partial y}=\bar{u}^{b} .
\end{gathered}
$$

(2) $m \geqslant 1$

$$
\begin{gathered}
\frac{\partial^{4} \hat{\psi}_{, m}}{\partial y^{4}}-2 \alpha^{2} \frac{\partial^{2} \hat{\psi}_{, m}}{\partial y^{2}}+\alpha^{4} \hat{\psi}_{, m}=0, \\
y=1: \hat{\psi}_{, m}(y)=0, \quad \frac{\partial \hat{\psi}_{, m}}{\partial y}=\hat{u}_{, m}^{t}, \\
y=-1: \hat{\psi}_{, m}(y)=0, \quad \frac{\partial \hat{\psi}_{, m}}{\partial y}=\hat{u}_{, m}^{b},
\end{gathered}
$$

where $\alpha=2 m \pi / L$. The subscript $(, m)$ stands for either $(s, m)$ or $(c, m)$. Equations (12) and (13) are linear and can thus be easily solved.

(1) $m=0$

$$
\bar{\psi}_{0}(y)=\frac{Q}{4}(2-y)(1+y)^{2}-\frac{1-y^{2}}{4}\left[\left(\bar{u}_{0}^{t}-\bar{u}_{0}^{b}\right)+y\left(\bar{u}_{0}^{t}+\bar{u}_{0}^{b}\right)\right]
$$

(2) $m \geqslant 1$

$$
\begin{aligned}
\hat{\psi}_{, m}(y)= & \frac{a_{, m}-b_{, m}}{2}(1-y) \sinh [\alpha(1+y)] \\
& -\frac{a_{, m}+b_{, m}}{2}(1+y) \sinh [\alpha(1-y)],
\end{aligned}
$$

where

$$
\begin{aligned}
& a_{, m}=\frac{\hat{u}_{, m}^{t}+\hat{u}_{, m}^{b}}{2 \alpha-\sinh (2 \alpha)}, \\
& b_{, m}=\frac{\hat{u}_{, m}^{t}-\hat{u}_{, m}^{b}}{2 \alpha+\sinh (2 \alpha)} .
\end{aligned}
$$

The zero mode of the stream function can be simplified by the substitution of Eq. (8) into Eq. (14),

$$
\bar{\psi}_{0}(y)=\frac{\bar{u}_{0}^{t}+\bar{u}_{0}^{b}}{2}(1+y)-\frac{1-y^{2}}{4}\left(\bar{u}_{0}^{t}-\bar{u}_{0}^{b}\right) \text {, }
$$

which gives the primary EOFs

$$
\bar{u}(y)=\frac{\bar{u}_{0}^{t}+\bar{u}_{0}^{b}}{2}+\frac{\bar{u}_{0}^{t}-\bar{u}_{0}^{b}}{2} y,
$$

$$
\bar{v}(y)=0 .
$$

It is easily seen from Eq. (18) that the primary EOF is a linear shear flow. The symmetric slip velocities on the boundary produce a plug flow, while the asymmetric slip velocities on the boundary yield a shear flow.

The nonzero modes of the stream function can be simplified if the variation of the wall charges in the $x$ direction is very slow such that the Fourier modes of larger wave numbers for the stream functions and the slip velocities can be ignored. In other words, the characteristic length scales of the variation are much smaller than the length scale $L$ of the microchannels. In this case, the number of the modes is small, such as $N \ll L / 2 \pi$ ( $N$ is the number of the Fourier modes with nonzero coefficients); then, $\alpha=2 m \pi / L$ is also very small. This assumption yields

$$
\begin{aligned}
\psi^{(s e c)}= & -\frac{(1+y)\left(1-y^{2}\right)}{4} \sum_{m=1}^{N}\left\{\hat{u}_{s, m}^{t} \sin \left(\frac{2 \pi m x}{L}\right)\right. \\
& \left.+\hat{u}_{c, m}^{t} \cos \left(\frac{2 \pi m x}{L}\right)\right\} \\
& +\frac{(1-y)\left(1-y^{2}\right)}{4} \sum_{m=1}^{N}\left\{\hat{u}_{s, m}^{b} \sin \left(\frac{2 \pi m x}{L}\right)\right. \\
& \left.+\hat{u}_{c, m}^{b} \cos \left(\frac{2 \pi m x}{L}\right)\right\} .
\end{aligned}
$$

Therefore, the velocity of the secondary flow is

$$
\begin{aligned}
u^{(s e c)}= & -\frac{(1-3 y)(1+y)}{4} \sum_{m=1}^{N}\left\{\hat{u}_{s, m}^{t} \sin \left(\frac{2 \pi m x}{L}\right)\right. \\
& \left.+\hat{u}_{c, m}^{t} \cos \left(\frac{2 \pi m x}{L}\right)\right\} \\
& -\frac{(1+3 y)(1-y)}{4} \sum_{m=1}^{N}\left\{\hat{u}_{s, m}^{b} \sin \left(\frac{2 \pi m x}{L}\right)\right. \\
& \left.+\hat{u}_{c, m}^{b} \cos \left(\frac{2 \pi m x}{L}\right)\right\},
\end{aligned}
$$

$$
\begin{aligned}
v^{(s e c)}= & \frac{(1+y)\left(1-y^{2}\right)}{4} \sum_{m=1}^{N} \frac{2 \pi m}{L}\left\{\hat{u}_{s, m}^{t} \cos \left(\frac{2 \pi m x}{L}\right)\right. \\
& \left.-\hat{u}_{c, m}^{t} \sin \left(\frac{2 \pi m x}{L}\right)\right\} \\
& -\frac{(1-y)\left(1-y^{2}\right)}{4} \sum_{m=1}^{N} \frac{2 \pi m}{L}\left\{\hat{u}_{s, m}^{b} \cos \left(\frac{2 \pi m x}{L}\right)\right. \\
& \left.-\hat{u}_{c, m}^{b} \sin \left(\frac{2 \pi m x}{L}\right)\right\} .
\end{aligned}
$$

The secondary flows in the EOF are completely determined by the nonzero modes in the slip velocities. The symmetric slip velocities, $\hat{u}_{s, m}^{t}=\hat{u}_{s, m}^{b}$ and $\hat{u}_{c, m}^{t}=\hat{u}_{c, m}^{b}$, yield the symmetric solution 


$$
\begin{aligned}
u^{(s e c)}= & -\frac{\left(1-3 y^{2}\right)}{2} \sum_{m=1}^{N} \frac{2 \pi m}{L}\left\{\hat{u}_{s, m}^{t} \sin \left(\frac{2 \pi m x}{L}\right)\right. \\
& \left.+\hat{u}_{c, m}^{t} \cos \left(\frac{2 \pi m x}{L}\right)\right\}, \\
v^{(s e c)}= & \frac{y\left(1-y^{2}\right)}{2} \sum_{m=1}^{N} \frac{2 \pi m}{L}\left\{\hat{u}_{s, m}^{t} \cos \left(\frac{2 \pi m x}{L}\right)\right. \\
& \left.-\hat{u}_{c, m}^{t} \sin \left(\frac{2 \pi m x}{L}\right)\right\},
\end{aligned}
$$

and the asymmetric slip velocities yield asymmetric solutions.

It can be seen from Eqs. (18) and (21) that the primary and secondary EOFs are dependent on slip velocities, or equivalently, zeta potentials. The primary EOFs are dependent on uniform slip velocities, where the symmetrically uniform slip velocities generate plug flows and the asymmetrically uniform slip velocities generate shear flows. The secondary EOFs are dependent on nonuniform slip velocities. The nonuniform slip velocity can generate various complex flow patterns. We will study the secondary EOFs generated by the wavelike zeta potentials in the next section.

\section{NUMERICAL SIMULATION OF EOF AND ITS MIXING}

The analytical solutions in the last section held for the EOFs at a very low Reynolds number, where the convection effect can be ignored. In this section, we will numerically solve the full Navier-Stokes equations with the slip velocity boundary conditions, and compare the numerical results with the analytical solutions.

The EOFs in the microchannel are shown in Fig. 1: the half-width of the channel is taken as unity, $\delta=1$, and the length of the channel $L=60 \delta$. The movement of EOFs in microchannels is described by a two-dimensional steady Navier-Stokes equation,

$$
\begin{aligned}
& u \frac{\partial u}{\partial x}+v \frac{\partial u}{\partial y}=-\frac{\partial p}{\partial x}+\frac{1}{\operatorname{Re}}\left(\frac{\partial^{2} u}{\partial x^{2}}+\frac{\partial^{2} u}{\partial y^{2}}\right) \\
& u \frac{\partial v}{\partial x}+v \frac{\partial v}{\partial y}=-\frac{\partial p}{\partial y}+\frac{1}{\operatorname{Re}}\left(\frac{\partial^{2} v}{\partial x^{2}}+\frac{\partial^{2} v}{\partial y^{2}}\right),
\end{aligned}
$$

and the mixing process of scalars, such as reagents and species, is described by a two-dimensional advection-diffusion equation,

$$
u \frac{\partial \phi}{\partial x}+v \frac{\partial \phi}{\partial y}=\frac{1}{\operatorname{Pe}}\left(\frac{\partial^{2} \phi}{\partial x^{2}}+\frac{\partial^{2} \phi}{\partial y^{2}}\right),
$$

where $u$ and $v$ are the streamwise and normal components of velocity field, respectively, and $\phi$ is a passive scalar. Re $=U \delta / \nu$ is the Reynolds number and $\mathrm{Pe}=U L / \kappa$ is the Peclet number, where $U=Q / 2 \delta$ is the mean velocity, $\nu$ dynamic viscosity, and $\kappa$ diffusivity. In microchannels, Re is usually small $(1<\operatorname{Re}<100)$ and Pe large $\left(10^{3}<\mathrm{Pe}<10^{5}\right)$. Here, we take $\mathrm{Re}=10$ and $\mathrm{Pe}=10^{4}$.
The periodic boundary conditions are taken for the velocity field in the streamwise direction, and the wavelike slip velocity boundary conditions on the surfaces will be given later in four different cases. To facilitate the investigation of mixing process, we consider the case of a scalar introduced into the microchannel at the inlet with uniform concentration in one half-domain and zero in another half-domain, such as

$$
\phi(x=0, y)=\left\{\begin{array}{lc}
2, & -1 \leqslant y<0 \\
0, & 0 \leqslant y<1,
\end{array}\right.
$$

and the scalar at the wall and outlet

$$
\frac{\partial \phi}{\partial \mathbf{n}}=0
$$

where the unity vector $\mathbf{n}$ is in the normal direction at the wall and in the streamwise direction at the outlet, respectively.

The SIMPLE algorithm [12], based on the finite volume method, are used to numerically solve Eqs. (22) and (23), where the computational domain is discretized into 2400 $\times 80$ uniform grids. In order to reduce the effect of inlet and exit boundary conditions, an extended channel of $10 \delta$ length is added both at the inlet and exit of the channel under consideration, respectively. The Navier-Stokes equations (22) are then solved to establish the velocity field in the entire domain.

We will first consider the symmetrically slip velocity boundary condition,

$$
u^{t}(x)=u^{b}(x)=1+4 \sin \left(\frac{2 \pi x}{L}\right) .
$$

Figure 2 shows the streamlines of secondary and total EOFs (primary plus secondary) in the boundary condition (26), where Figs. 2(a) and 2(c) are the plots for the secondary and total flows from direct numerical simulation (DNS) and Figs. 2(b) and 2(d) are the plots for the ones from analytical solutions. The secondary EOFs consist of the two noninteracting, symmetrically recirculating rolls. The total EOFs are the symmetric pattern of recirculating rolls and locally stretched streamlines.

We will next consider that the slip velocities at the top and bottom surfaces have different amplitudes,

$$
\begin{aligned}
& u^{t}(x)=1+4 \sin \left(\frac{2 \pi x}{L}\right), \\
& u^{b}(x)=1+8 \sin \left(\frac{2 \pi x}{L}\right) .
\end{aligned}
$$

Figure 3 shows the streamlines of secondary and total FOFs in the boundary conditions (27) in the same way as Fig. 2. The secondary flows consist of larger recirculating rolls and smaller ones. These two rolls in the normal direction are not interacting. The total flows consist of asymmetric recirculating rolls and separation streamlines. The separation line from left to right in the numerical simulation do not appear in the analytical solution due to the small viscosity.

The third case is that the slip velocities at the top and bottom surfaces have different phases, 

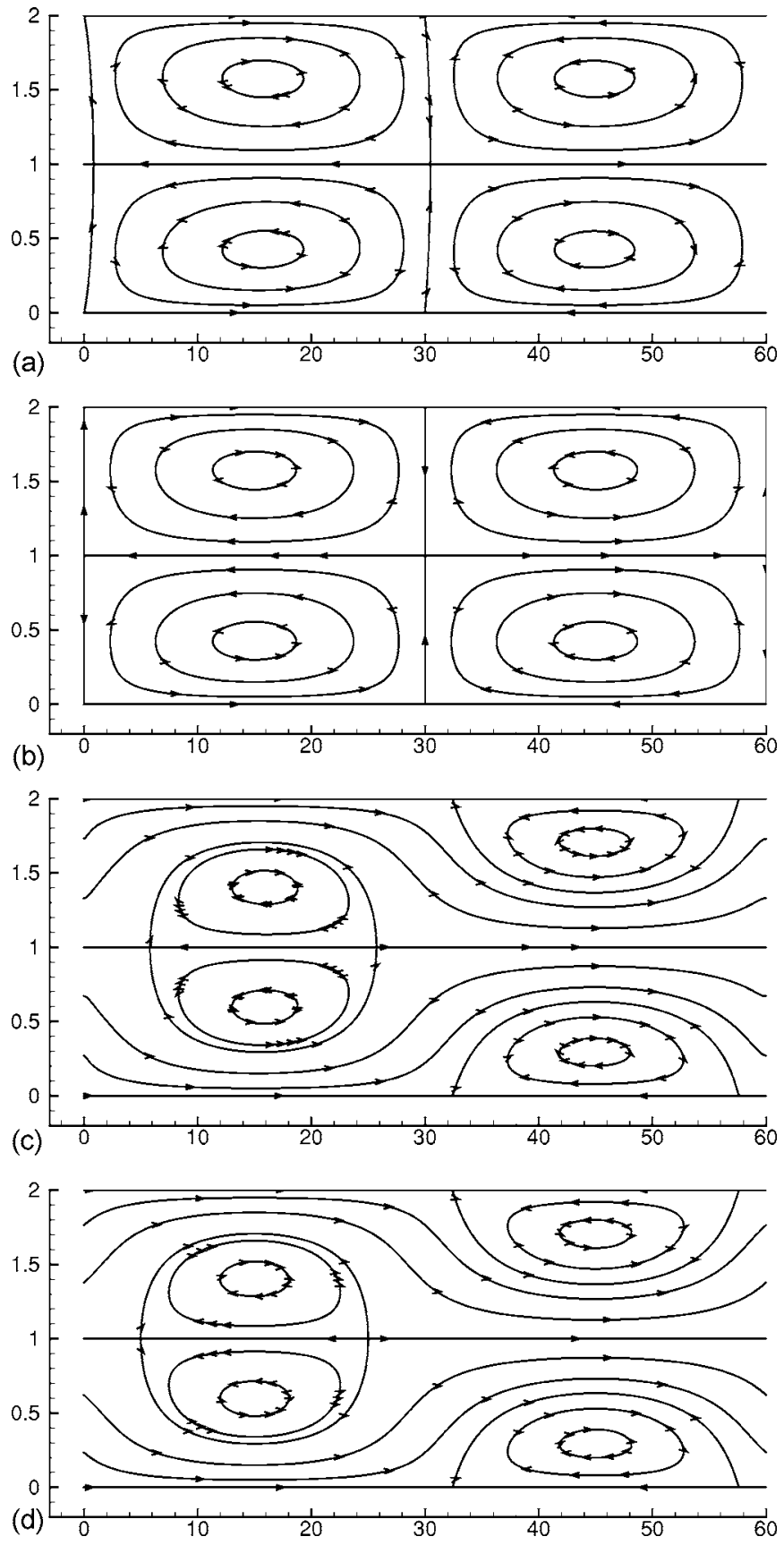

FIG. 2. The streamlines of EOFs generated by symmetrically wavelike zeta potentials: (a) secondary EOFs from DNS; (b) secondary EOFs from analytical solutions; (c) total flows from DNS; (d) total flows from analytical solutions.

$$
\begin{gathered}
u^{t}=1+4 \sin \left(\frac{2 \pi x}{L}\right), \\
u^{b}=1+4 \sin \left(\frac{2 \pi x}{L}+\pi\right) .
\end{gathered}
$$

Figure 4 shows the streamlines of secondary and total EOFs in the boundary condition (28) in the same way as in Fig. 2. The secondary EOFs exhibit the parallel antirotational rolls. The streamlines from the top surface to the bottom surface
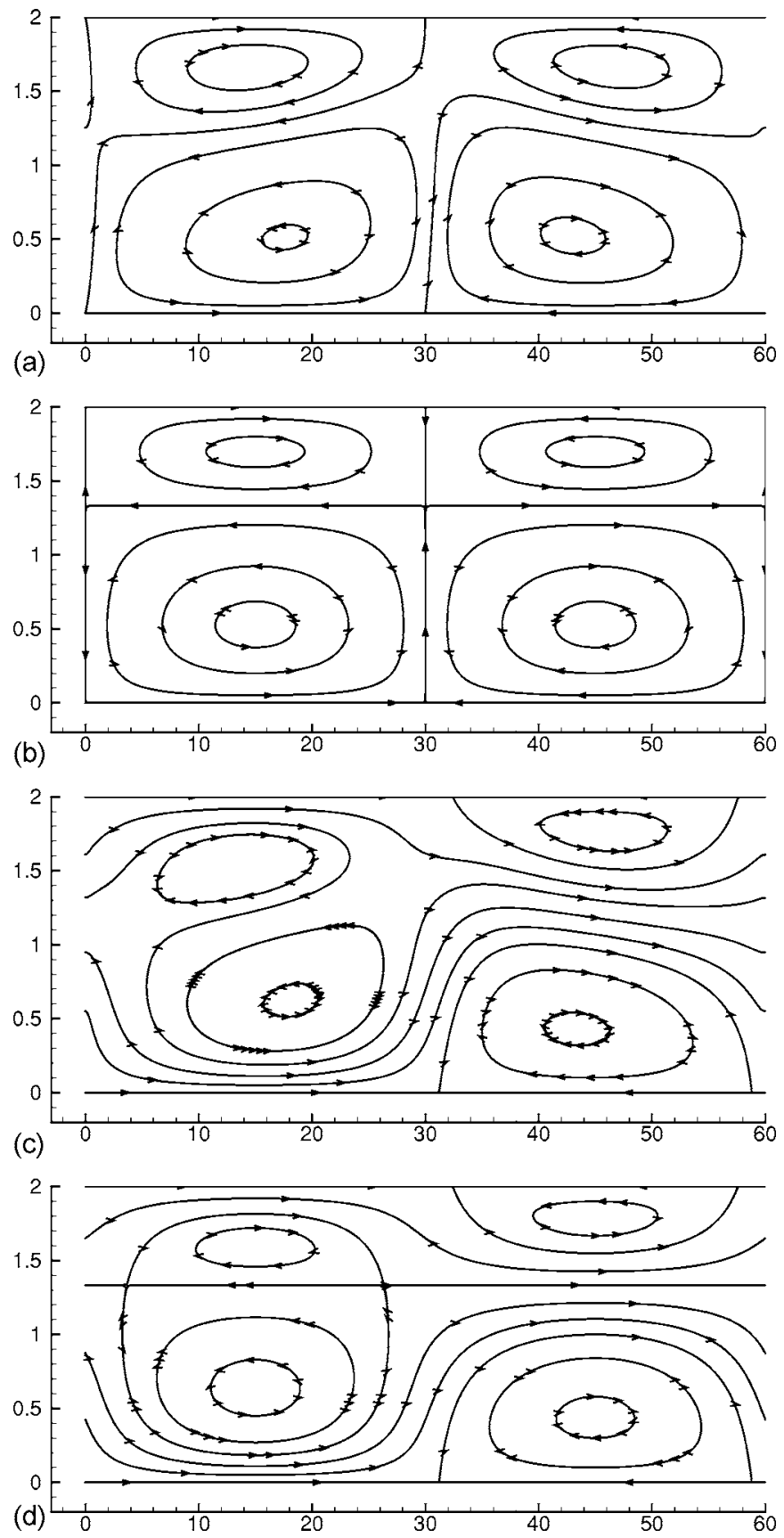

FIG. 3. The streamlines of EOFs generated by asymmetrcally wavelike zeta potentials with different amplitudes: (a) secondary EOFs from DNS; (b) secondary EOFs from analytical solutions; (c) total flows from DNS; (d) total flows from analytical solutions.

represent the effects of phase difference in slip velocity boundary conditions. The total flows are the sum of the antirotational rolls and separation streamlines.

The final case is that the slip velocities at the top and bottom surfaces have different wave numbers,

$$
u^{t}=1+4 \sin \left(\frac{2 \pi x}{L}\right)
$$



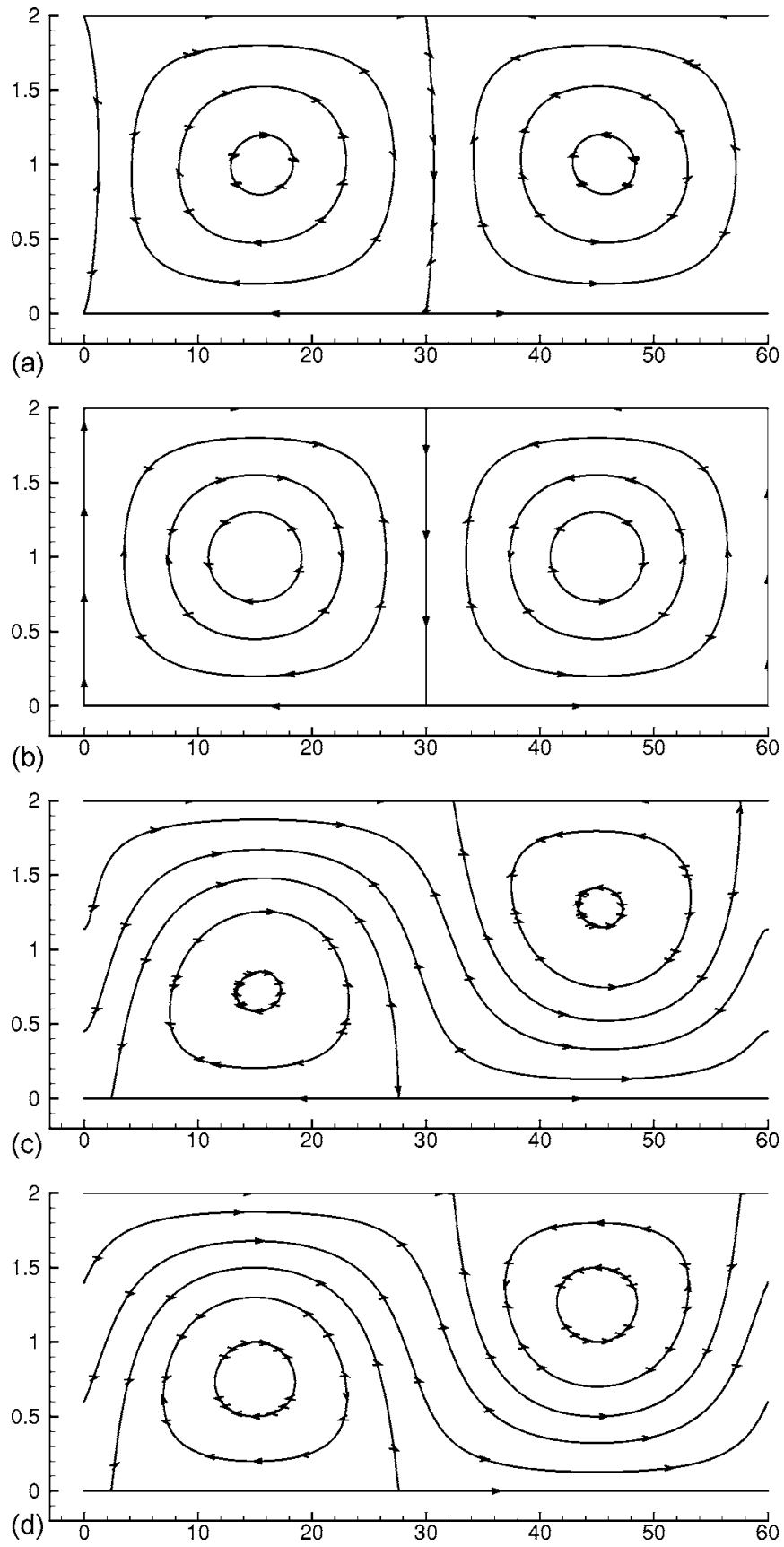

FIG. 4. The streamlines of EOFs generated by asymmetrically wavelike zeta potentials with different phases: (a) secondary EOFs from DNS; (b) secondary EOFs from analytical solutions; (c) total flows from DNS; (d) total flows from analytical solutions.

$$
u^{b}=1+4 \sin \left(\frac{4 \pi x}{L}\right)
$$

Figure 5 shows the secondary and total EOFs in the same way as in Fig. 2. The secondary flows consist of both local and cross-sectional rolls. The total EOFs exhibit complex flow patterns: saddle points, rolls, and separation streamlines. There are small differences between the analytical and numerical solutions at the inlet and outlet.
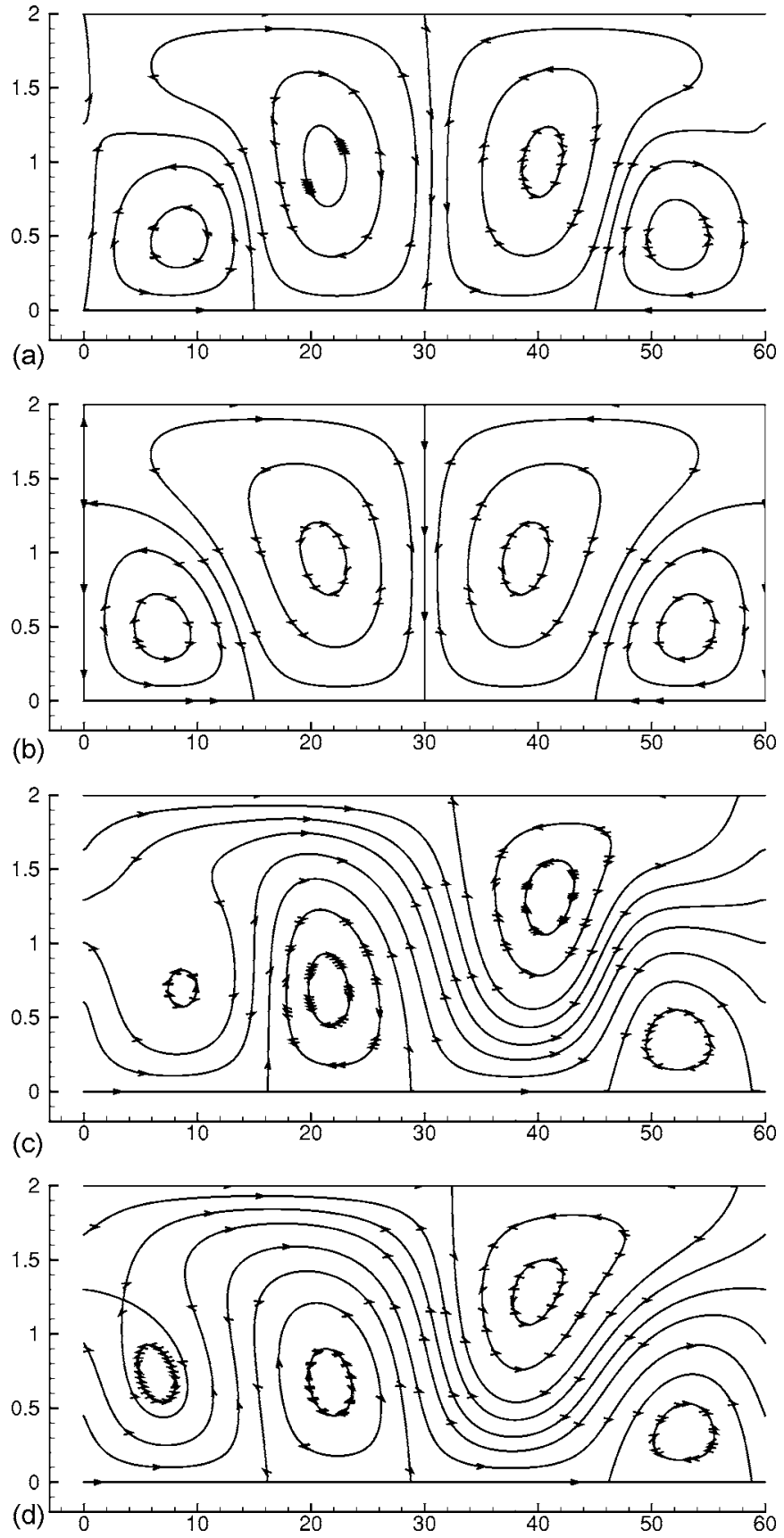

FIG. 5. The streamlines of EOFs generated by asymmetrically wavelike zeta potentials with different wave numbers: (a) secondary EOFs from DNS; (b) secondary EOFs from analytical solutions; (c) total flows from DNS; (d) total flows from analytical solutions.

Primary EOFs are generated by constant zeta potentials, either symmetrically or asymmetrically. The latter is a linear shear flow. Secondary EOFs are generated by wavelike zeta potentials. They exhibit larger transversal advections which could significantly enhance scalar mixing. It is also shown from Figs. 2-5 that the analytical solutions obtained in the last section are mostly in agreement with the numerical results, where the numerical results show a slight bulge toward increasing $x$. Therefore, the Stokes approximation here for EOFs is valid. 


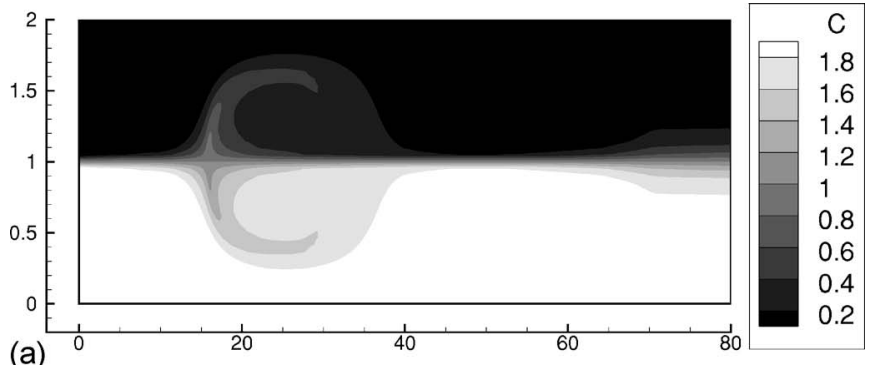

(a)
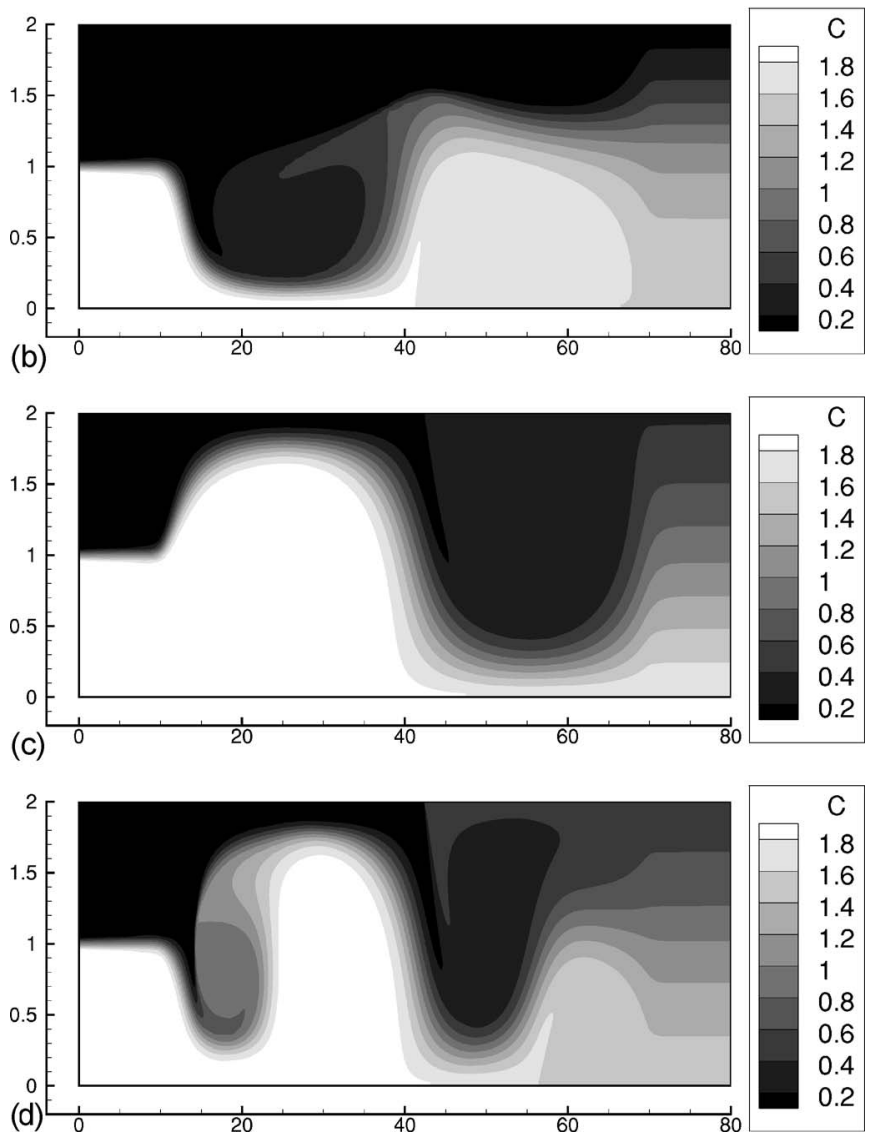

FIG. 6. The scalar gray-scale contours in the EOFs: (a) symmetrically wavelike zeta potentials; (b) asymmetrically wavelike zeta potentials with different amplitudes; (c) asymmetrically wavelike zeta potentials with different phases; (d) asymmetrically wavelike zeta potentials with different wave numbers.

Figure 6 shows the scalar gray-scale contours in the four different cases of EOFs as indicated in Eqs. (26)-(29). These plots provide the pictures of how the mixing of two different reagents at the inlet progresses in the EOFs. Compared with Figs. 2-5, Fig. 6 indicates that the secondary EOFs could transport the scalars more strongly than the primary ones. The transportation increases the magnitudes of scalar gradients in the larger areas and enhance the scalar mixing.

In order to further characterize the mixing capability of EOFs, we plot the scalar profiles at the outlet in Fig. 7. For comparison, we also plot the scalar profile in the plug flows, where the symmetrically constant slip velocities are imposed on the boundary conditions. The mixing capability can be evaluated by the approaches of the scalar profiles to the fully mixing region $\phi=1$. Obviously, the plug flows induces little

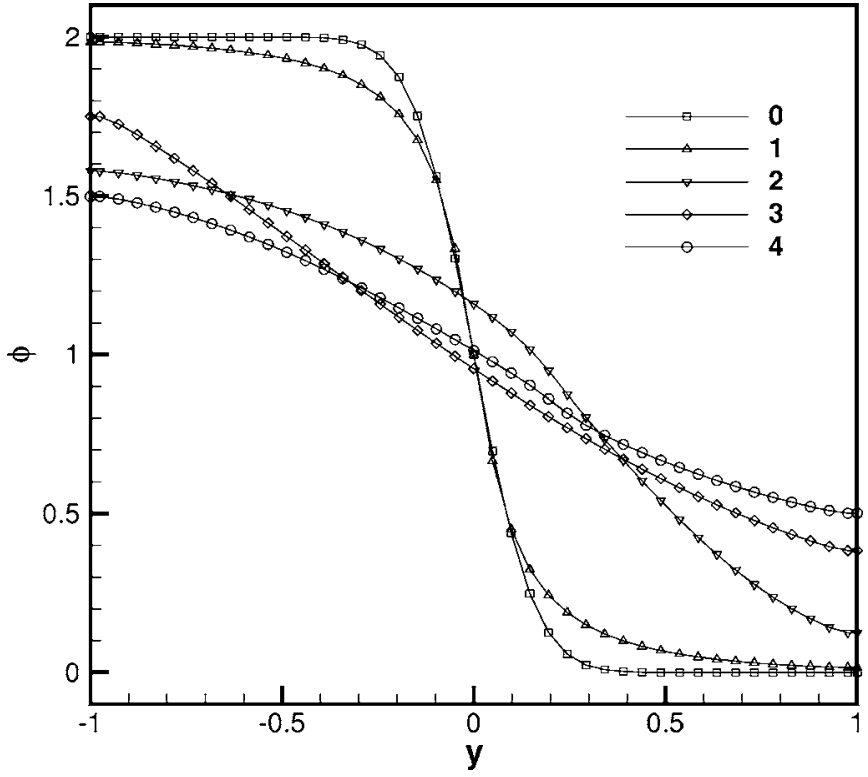

FIG. 7. The scalar profile at the outlet: the number 0 (square) denotes the symmetrically uniform zeta potentials; the number 1 (upper triangle) denotes the symmetrically wavelike zeta potentials; the number 2 (down triangle) denotes the asymmetrically wavelike zeta potentials with different amplitudes; the number 3 (skew square) denotes the asymmetrically wavelike zeta potentials with different phases; the number 4 (circle) denotes the asymmetrically wavelike zeta potentials with different wave numbers.

mixing and the symmetric slip velocity boundary induces small mixing. The heterogeneous slip velocity boundary has more efficient mixing than symmetric ones: the slip velocity boundary with the different wave number is the strongest; the slip velocities with the different amplitude and the different phase have comparable capabilities to enhance the mixing rate.

\section{CONCLUDING REMARKS}

EOF and its mixing in microchannels with heterogeneous zeta potentials are studied in this paper. The results show that the heterogeneous zeta potentials could generate complex flow patterns: the symmetrically wavelike slip zeta potentials could generate symmetrically secondary EOFs and enhance scalar mixing; the wavelike zeta potentials with either different amplitudes or phases could generate asymmetrical rolls and improve the mixing rate of scalars; the wavelike zeta potentials with different wave numbers could generate the asymmetrically recirculating flows and significantly enhance scalar mixing. The mechanism of the mixing enhancement is that the advection of secondary EOFs increases scalar gradients and, simultaneously, the molecular diffusion makes faster mixing of the scalar where its gradients are larger. The analysis and numerical simulations in the present paper are based on the two-dimensional flows with the large aspect ratios, which may not be generally valid for the microchannels with nearly square cross sections. However, it is still true that the heterogeneous zeta potentials could generate more complex flow patterns and enhance the mixing in threedimensional EOFs. 


\section{ACKNOWLEDGMENTS}

G. W. He is grateful to Dr. H. Lin and Dr. Z. F. Zhang for valuable conversations and correspondence. This work was supported by the Chinese Academy of Sciences under the innovative project "Multi-scale modelling and simulation in complex system" (KJCX-SW-L08) and National Natural Science Foundation of China under the Project No. 10325211.
[1] H. A. Stone, A. D. Strook, and A. Ajdari, Annu. Rev. Fluid Mech. 36, 381 (2004).

[2] R. Probstein, Physicochemical Hydrodynamics (Wiley, New York, 1994).

[3] J. L. Anderson and W. K. Idol, Chem. Eng. Commun. 38, 93 (1985).

[4] S. Ghosal, J. Fluid Mech. 459, 103 (2002).

[5] A. E. Herr, J. I. Molho, J. G. Santiago, M. G. Mungal, T. W. Kenny, and M. G. Garguilo, Anal. Chem. 72, 1053 (2000).

[6] A. D. Stroock, M. Weck, D. T. Chiu, W. T. S. Huck, P. J. A. Kenis, R. F. Ismagilov, and G. M. Whitesides, Phys. Rev. Lett.
84, 3314 (2000).

[7] A. Ajdari, Phys. Rev. Lett. 75, 755 (1995).

[8] A. Ajdari, Phys. Rev. E 53, 4996 (1996).

[9] A. D. Strook, S. K. W. Dertinger, A. Ajdari, I. Mezic, H. A. Stone, and G. M. Whitesides, Science 295, 647 (2002).

[10] T. J. Johnson, D. Rose, and L. E. Locascio, Anal. Chem. 74, 45 (2002)

[11] H. Aref, Phys. Fluids 14, 1315 (2002).

[12] S. V. Patankar, Numerical Heat Transfer and Fluid Flow (Hemisphere Publishing Cor., 1980). 